\title{
Misregulation of Gene Expression in the Redox-Sensitive NF-kB-Dependent Limb Outgrowth Pathway by Thalidomide
}

\author{
JASON M. HANSEN, ${ }^{1}$ SIEW-GING GONG, ${ }^{2}$ MARTIN PHILBERT, ${ }^{1}$ AND CRAIG HARRIS ${ }^{1 *}$ \\ ${ }^{1}$ Toxicology Program, Environmental Health Sciences, School of Public Health, University of Michigan, \\ Ann Arbor, Michigan \\ ${ }^{2}$ Orthodontic and Pediatric Dentistry, School of Dentistry, University of Michigan, Ann Arbor, Michigan
}

\begin{abstract}
Thalidomide is known to induce oxidative stress, but mechanisms have not been described through which oxidative stress could contribute to thalidomide-induced terata. Oxidative stress modulates intracellular glutathione (GSH) and redox status and can perturb redox-sensitive processes, such as transcription factor activation and/or binding. Nuclear factorkappa $B(N F-\kappa B)$, a redox-sensitive transcription factor involved in limb outgrowth, may be modulated by thalidomide-induced redox shifts. Thalidomide-resistant Sprague-Dawley rat embryos (gestation day [GD] 13) treated with thalidomide in utero showed no changes in GSH distribution in the limb but thalidomide-sensitive New Zealand White rabbit embryos (GD 12) showed selective GSH depletion in the limb bud progress zone (PZ). NF- $\mathrm{B}$ and regulatory genes that initiate and maintain limb outgrowth and development, such as Twist and Fgf-10, are selectively expressed in the PZ. Green fluorescent protein (GFP) reporter vectors containing NF-кB binding promoter sites were transfected into both rat and rabbit limb bud cells (LBCs). Treatment with thalidomide caused a preferential decrease in GFP expression in rabbit $\mathrm{LBCs}$ but not in rat LBCs. N-acetylcysteine and $\alpha$-N-t-phenylbutyl nitrone (PBN), a free radical trapping agent, rescued GFP expression in thalidomide-treated cultures compared with cultures that received thalidomide only. In situ hybridization showed a preferential decrease in Twist, Fgf-8, and Fgf-10 expression after thalidomide treatment $(400$ $\mathrm{mg} / \mathrm{kg}$ per day) in rabbit embryos. Expression in rat embryos was not affected. Intravenous cotreatment with PBN and thalidomide (gavage) in rabbits restored normal patterns and localization of Twist, Fgf-8, and Fgf-10 expression. These findings show that NF-kB binding is diminished due to selective thalidomide-induced redox changes in the rabbit, resulting in the significant attenuation of expression of genes necessary for limb outgrowth. $\odot 2002$ Wiley-Liss, Inc.
\end{abstract}

Key words: thalidomide; NF-кB; redox status; oxidative stress; limb; teratogenesis

\section{INTRODUCTION}

Mechanisms underlying the production of functional and anatomic developmental abnormalities are difficult to describe due to the combined effects of chemical and environmental factors upon an already complex developmental program. The etiologies of most diseases with early developmental origins are mostly unknown, but many are postulated to be multifactorial, involving both genetic and environmental components. A definition of the biological interface between these genetic events and the suspected environmental factors has been elusive. An important recent advance in this area has been the recognition that changes in the balance of oxidizing and reducing equivalents (redox status) within the cell can have profound regulatory consequences in terms of the control of cell proliferation, differentiation, apoptosis, and several functional/ biochemical pathways. Signal transduction components, kinases, nuclear transcription factors, receptors, and transporters have all been shown to be sensitive to even relatively small changes in the intracellular redox potential. Critical to the discussion of development and disease in terms of the environmental interface is the well established fact that a common consequence of many environmental and chemical insults is a shift in the intracellular redox environment, particularly when the insult results in the generation of free radicals and/or excessive reactive oxygen species (ROS). These events are due primarily to the oxidation of soluble and protein sulfhydryls such as glutathione (GSH) and the free cysteine residues of proteins (protein-glutathione mixed disulfides; prot-SSG) to protect vital cellular components and restore reducing conditions. The GSH redox status in developing cells may be the critical environmental sensor that mediates the exchange of regulator information between the environment and the genome. Our recent and ongoing characterizations of the effects of environmental and therapeutic chemi-

*Correspondence to: Craig Harris, Department of Environmental Health Sciences, SPH II M6116, 1420 Washington Heights, Ann Arbor, MI 48109-2029. E-mail: charris@umich.edu

Received 26 November 2001; Accepted 22 July 2002

DOI $10.1002 /$ dvdy.10150 
cals on the embryonic GSH status have led to the suggestion that temporal, spatial, and species differences in sensitivity to chemical exposure is related to a cell's inherent ability to maintain and regulate its intracellular GSH and redox status within a range that is permissive for normal signaling and regulation (Hansen et al., 2001a). Several human and animal teratogens are known to produce free radicals or other reactive chemical intermediates as part of their biotransformation and mechanism of teratogenesis. We support the concept that the resulting disruption of the normal redox environment causes the misregulation of gene expression, contributing to malformation and disease. Previous work has demonstrated that the human teratogen thalidomide causes oxidative stress and ROS production (Hansen et al., 1999; Parman et al., 1999; Sauer et al., 2000). This communication reports how thalidomide, through its ability to alter redox status, exerts selective changes in the regulation of limb outgrowth in the sensitive rabbit but not the resistant rat by misregulation of gene expression mediated through the redox-sensitive transcription factor, nuclear factor kappa B (NF-kB).

Numerous stimuli, including conditions of oxidative stress and ROS, can cause the dissociation of the cyto-

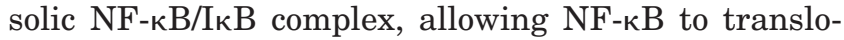
cate into the nucleus where it binds to its DNA recognition sequence (5'GGGACTTTCC'3) and initiates gene transcription. NF-кB binding to DNA is dependent on the reduced state of a critical cysteine residue $\left(\right.$ cysteine $^{62}$ ) on the p50 subunit. Site-directed mutagenic substitution of cysteine ${ }^{62}$ with serine reduces the effectiveness of DNA binding and renders NF- $\mathrm{BB}$ inactive and insensitive to redox regulation (Matthews et al., 1992; Hayashi et al., 1993; Mitomo et al., 1994). The NF- $\mathrm{KB}$ regulation of gene expression in the limb bud has been described previously by Bushdid et al. (1998) and Kanegae et al. (1998), who concluded that normal NF-кB signaling is critical for limb bud outgrowth and development.

Establishment of a feedback loop between signals from the apical ectodermal ridge (AER) and signals from the progress zone (PZ), highly proliferative cells underlying the AER, is required to initiate and maintain limb outgrowth. Perturbations in normal signaling may result in the loss of proper gene activation and result in limb reduction. I- $\kappa \mathrm{B}$ mutant experiments that decrease the amount of translocated NF- $\mathrm{B}$ available for DNA binding caused the decreased expression of critical genes in the $\mathrm{PZ}$ and indirectly caused the decreased expression of genes in the AER and zone of polarizing activity (ZPA). Twist expression in the PZ, Fgf-8 expression in the AER, and Shh in the ZPA were all affected by the disruption of normal NF-кB activities (Bushdid et al., 1998; Kanegae et al., 1998).

The TWIST gene product has been implicated in human and animal limb development. Mutations in this gene result in Saethre-Chotzen syndrome, which is characterized by craniofacial and limb malformations
(Bourgeois et al., 1998; Paznekas et al., 1998; Rice et al., 2000). The Twist protein contains a basic helixloop-helix motif, suggesting that it may act as a transcription factor. It is expressed in the limb in the area of the PZ and stimulates the expression of certain FGF receptors, namely Fgfr-1 and Fgfr-2 (Paznekas et al., 1998; Rice et al., 2000). Fgfr-2-deficient mice have been shown to have limb defects in which the affected limb bears a striking resemblance to thalidomide-induced limb malformations (Xu et al., 1998).

Fibroblast growth factor 10 (Fgf-10), like Twist, is expressed in the limb bud PZ. Fgf-10 has been shown to diffuse from the PZ and directly promote the expression of Fgf-8, which, in the limb, is found only in the AER (Ohuchi et al., 1997). This interaction between Fgf-10 in the progress zone and Fgf-8 in the AER is considered crucial for normal limb outgrowth (Crossley et al., 1996). The removal of the AER stunts limb outgrowth due to the loss of Fgf-8 signaling (Summerbell, 1974), but the addition of exogenous Fgf- 8 restores outgrowth (Vogel et al., 1996). Similarly, Fgf-10-deficient mice had no evidence of the formation of any limb elements (Kato and Sekine, 1999). The feedback loop that occurs between Fgf-10 and Fgf-8 is mediated in part by the Twist-regulated expression of an FGF receptor, Fgfr-2 (Xu et al., 1998). Perturbation of the interaction between $\mathrm{Fgf}-10$ and $\mathrm{Fgf}-8$ and compounded by decreased Twist expression results in faulty limb outgrowth, and amelia ensues. Besides phocomelia, amelia was one of the more common thalidomide-induced terata (Fabro, 1981).

Although our efforts in this project will concentrate on redox misregulation of NF- $\mathrm{kB}$-initiated pathways in the PZ and AER in the developing limb, thalidomide experiments are designed to specifically address both $\mathrm{NF}-\kappa \mathrm{B} / \mathrm{DNA}$ binding and the expression of NF- $\kappa \mathrm{B}-\mathrm{de}-$ pendent genes. Redox misregulation of $N F-\kappa B$ as a mechanism of teratogenesis may not only serve as a model for thalidomide teratogenicity but may also provide a general paradigm for the study of other teratogens that cause oxidative stress.

\section{RESULTS \\ Mercury Orange Histochemistry}

Mercury orange $(\mathrm{HgO})$ is a stain that permits the visualization of small biothiols, namely GSH and cysteine, which both serve as intracellular reducing equivalents and are important contributors to redox status. Loss of staining is indicative of a loss of GSH and suggests oxidative stress. Limb cryosections stained with $\mathrm{HgO}$ provide spatial information of thalidomideinduced oxidative stress in treated and control limbs, but not between species.

$\mathrm{HgO}$ staining of control rat limbs showed a relatively even distribution of thiols throughout the limb (Fig. 1). Treatment with $300 \mathrm{mg}$ of thalidomide/kg per day in utero did not produce any significant differences in rat embryonic limbs; a uniformity of mercury orange staining was maintained. 

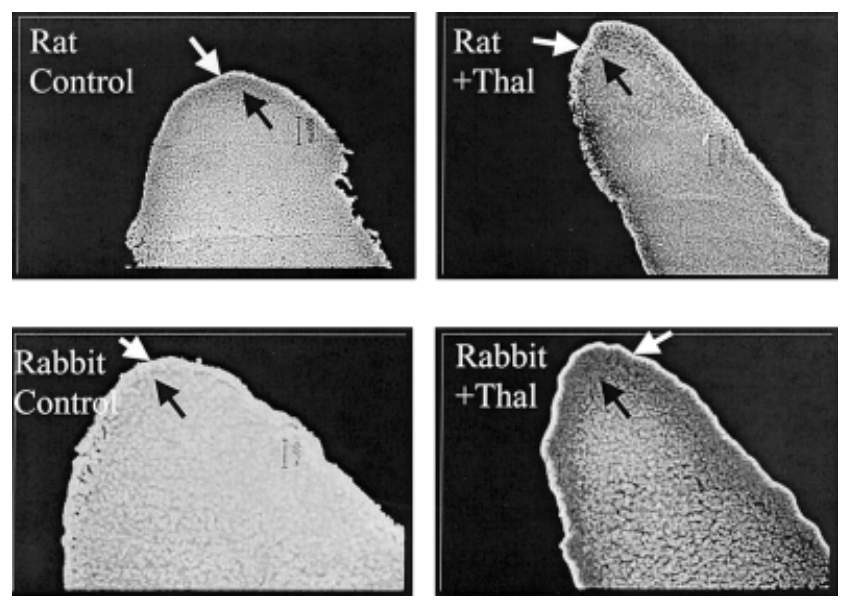

Fig. 1. Mercury orange $(\mathrm{HgO})$-stained rat (gestation day [GD] 13) and rabbit (GD 12) limbs treated with 300 and $70 \mathrm{mg}$ thalidomide/kg per day, respectively. Black arrows denote the region of the progress zone (PZ); and white arrows denote the area of the apical ectodermal ridge (AER). Rat thalidomide-treated limb buds did not show any relevant decrease in $\mathrm{HgO}$ staining compared with control rat limb buds, suggesting little or no oxidative stress. In thalidomide-treated rabbit limb buds, there is a clear decrease in $\mathrm{HgO}$ fluorescence in the $\mathrm{PZ}$ but not the overlying AER compared with control rabbit limb buds, indicating a depletion of $\mathrm{PZ}$ glutathione and regional oxidative stress.

Thiols were evenly distributed throughout control rabbit limbs (Fig. 1), but limbs from rabbit embryos treated with $70 \mathrm{mg}$ of thalidomide/kg/day showed a specific decrease in mercury orange staining in the limb bud PZ. Thiol status in the AER was unaffected in both rat and rabbit limbs after thalidomide treatment.

\section{pNF-кB-d2EGFP Transfection}

Because of the role of NF-кB as a critical regulator of limb development, the use of a reporter vector provides additional information on the effects of thalidomide directly on the DNA binding efficiency in intact LBCs. Furthermore, using an in vitro approach allows for treatment with specific pharmacologic agents that affect redox status in different ways. $\mathrm{N}$-acetylcysteine (NAC) is a cysteine precursor that, during periods of oxidative stress, provides the rate-limiting amino acid required for de novo GSH synthesis. Glutathione repletion restores intracellular redox status to normal reducing conditions. The addition of $\alpha$-phenyl-N-t-butylnitrone $(\mathrm{PBN})$, a free radical trapping agent, removes ROS before redox status is altered. Thus, the use of NAC and PBN promotes the restoration of normal intercellular redox status, albeit by different mechanisms.

Rat and rabbit LBCs transfected with pNF-кBd2EGFP treated with diamide exhibited significantly decreased GFP expression: $86 \%( \pm 2.1 \%)$ and $52 \%$ $( \pm 10.8 \%)$ of control fluorescence, respectively (Fig. 2). Thalidomide treatment of rat limb bud cells transfected with pNF-кB-d2EGFP did not result in fluorescence differences compared with control cultures (Fig.

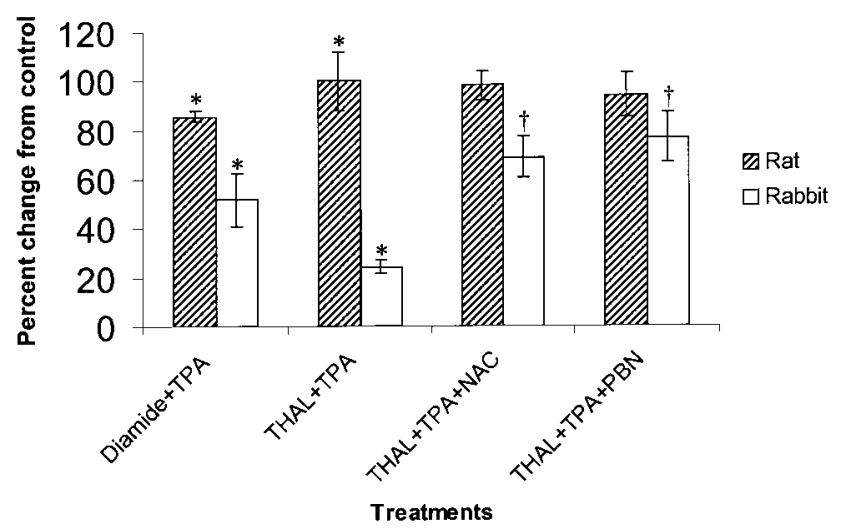

Fig. 2. pNF-кB-d2EGFP-transfected rat and rabbit limb bud cells show a decrease in GFP fluorescence with thalidomide treatment but are rescued by redox modulating agents, $\mathrm{N}$-acetylcysteine (NAC) and $\alpha$-phenyl-N-t-butylnitrone (PBN). Diamide, an oxidant and known inhibitor NF$\kappa B / D N A$ binding, acted as a positive control. Data are represented as percentage of control GFP expression: hatched bars denote transfected rat limb bud cells, and open bars denote transfected rabbit limb bud cells. Asterisks $\left({ }^{*}\right)$ denote a statistically significant $(<0.05)$ difference from control GFP expression. Crosses $(\dagger)$ represent a statistically significant $(<0.05)$ difference between transfected cells treated with thalidomide (THAL) and thalidomide + NAC or +PBN. TPA, tetradecanoylphorbol acetate.

2). However, rabbit LBCs transfected with pNF-кBd2EGFP and treated with $100 \mu \mathrm{M}$ thalidomide exhibited a significant decrease in GFP expression measuring $24 \%( \pm 2.8 \%)$ of the fluorescence seen in control cultures.

Pretreatment with NAC did not affect GFP expression in rat LBCs transfected with pNF-кB-d2EGFP (Fig. 2). However, thalidomide treatment effects on GFP expression in rabbit LBCs transfected with pNF$\kappa \mathrm{B}-\mathrm{d} 2 \mathrm{EGFP}$ could be partially rescued with NAC pretreatment. Expression of GFP was significantly increased with NAC treatment to $68 \%( \pm 8.9 \%)$ of control fluorescence compared with transfected cells receiving thalidomide only ( $24 \%$ of control values).

Pretreatment with the free radical trapping agent PBN had little effect on rat limb bud cells but proved very protective in rabbit limb bud cells. GFP fluorescence was increased from $24 \%$ in rabbit LBCs treated with thalidomide only to $77 \%( \pm 10.2 \%)$ in rabbit $\mathrm{LBCs}$ treated with both thalidomide and PBN (Fig. 2). Although PBN treatments were slightly more protective than NAC, differences were not significant.

\section{Whole-Mount In Situ Hybridization}

Comparison of gene expression between species was performed in situ for genes that have been shown previously to be affected by the disruption of normal NF-кB activity. Specifically, Fgf-10, Twist, and Fgf-8 were evaluated in embryos from early organization of limb outgrowth (GD 11.5 rat, GD 10 rabbit) to later stages of limb development (GD 13 rat; GD 11 and 12 rabbit). 

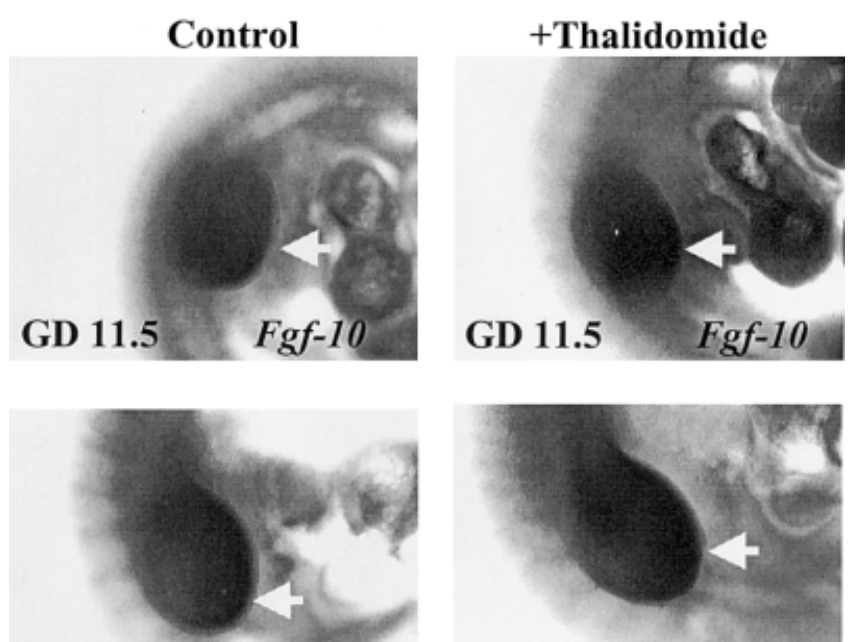

GD 11.5 Twist

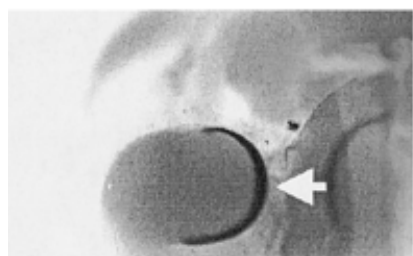

GD 11.5 $F g f-8$
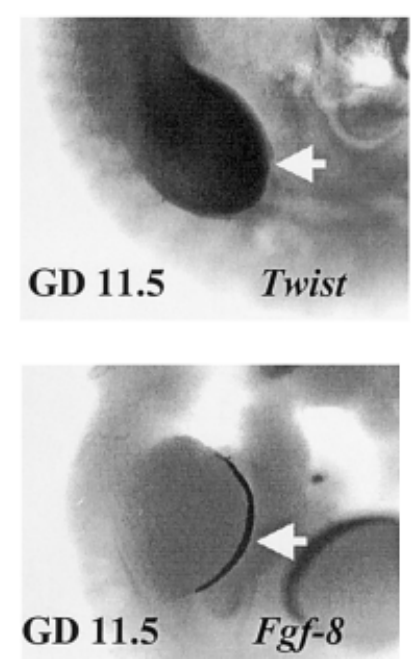

Fig. 3. In situ hybridization of control and thalidomide-treated rat embryos for Fgf-10, Twist, and Fgf-8 expression in the gestation day (GD) 11.5 rat limb bud. Thalidomide treatment had no effect on the expression of Fgf-10, Twist, and Fgf-8 compared with control. Arrows denote the limb and regions where Fgf-10, Twist, and Fgf-8 expression normally occurs.

Localization and expression of Fgf-10 was found throughout the PZ of control rat embryos on GD 11.5 and 13, the normal region of Fgf-10 expression in the limb (Figs. 3, 4). Fgf-10 expression in GD 11.5 rat embryonic limbs was spread throughout the presumptive limb bud field. More-developed limb buds from GD 13 rat embryos showed a more easily detectable pattern of Fgf-10 expression localized in the PZ (Fig. 4). Basically, the localization and expression of Fgf-10 in the rat embryonic limb (GD 11.5 or 13) was not altered with thalidomide treatment and was similar to control limb bud patterns of Fgf-10 localization and expression. Rat limb morphology, in both size and symmetry, was not affected on either GD 11.5 or GD 13 with thalidomide treatment and appeared similar to control embryonic limbs.

Control rat limb buds from GD 11.5 and 13 embryos showed localization and expression of Twist not only in the limb bud PZ but also throughout the entire limb bud mesenchymal field. Similar to Fgf-8 and Fgf-10 expression, no significant alterations in Twist expression were observed in GD 11.5 or $13 \mathrm{limb}$ buds from rat embryos receiving thalidomide treatment and appeared identical to control limb buds (Figs. 3, 4). Sim-
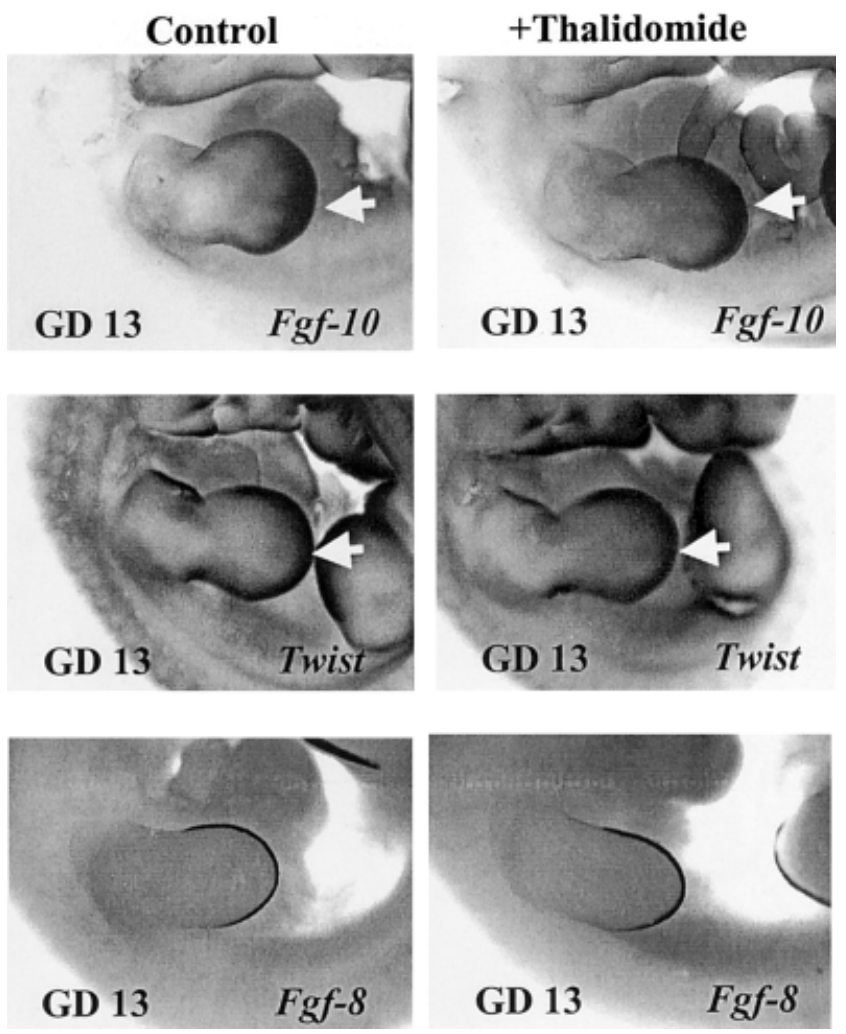

Fig. 4. In situ hybridization of control and thalidomide-treated rat embryos for Fgf-10, Twist, and Fgf-8 expression in the gestation day (GD) 13 rat limb bud. Thalidomide treatment had no effect on the expression of Fgf-10, Twist, and Fgf-8 compared with control. Arrows denote regions where Twist expression normally occurs.

ilar to $F g f-10$ probed embryos, rat limb morphology was not affected by thalidomide treatment and seemed equivalent in size, shape, and symmetry.

Control rat embryos showed Fgf-8 localization and expression in the AER on GD 11.5 and 13 across the limb bud periphery, the normal region for $F g f-8$ expression (Fig. 5). Thalidomide treatment did not affect the expression or localization of Fgf-8 in the AER on either GD 11.5 or 13 (Figs. 3, 4). No abnormalities were observed in rat limb buds treated with thalidomide compared with control rat limb buds.

The expression and localization of $\mathrm{Fgf-10}$ in control rabbit embryos was normal in GD 10 limbs confined to the region of the presumptive PZ (Fig. 5) Fgf-10 localization was more easily assessed in rabbit limb buds that had progressed further in development on GD 11 and 12. Thalidomide treatment caused a decrease in expression of Fgf-10 in rabbit limb buds from GD 10, 11 , and 12 embryos. In younger embryos (GD 10 and 11), Fgf-10 expression was greatly attenuated and almost undetectable. Expression of Fgf-10 in GD $12 \mathrm{em}-$ bryos was restricted to the more proximal and lateral portions of the limb and was not found in the distal tip of the limb bud, the region of the PZ, where it is notably absent. Although difficult to evaluate on GD 10, GD 11, 

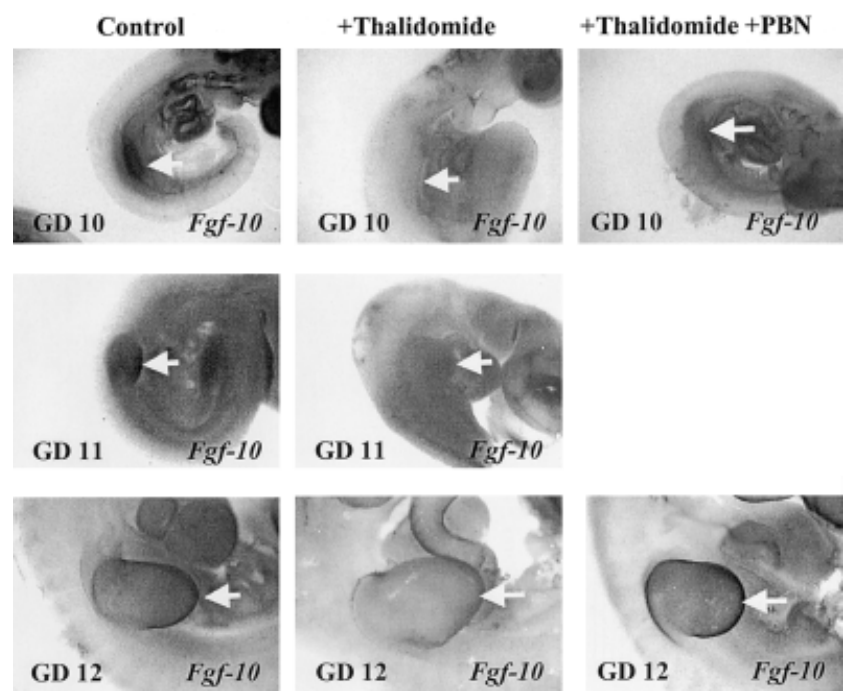

Fig. 5. In situ hybridization of control, thalidomide-treated, and thalidomide $+\alpha$-phenyl-N-t-butylnitrone (PBN) rabbit embryos for Fgf-10 expression in the gestation day (GD) 10-12 rabbit limb bud. Thalidomide greatly reduced the expression of $F g f-10$ at each stage of limb development. However, gene expression was not affected in embryos that received both thalidomide and the free radical trapping agent, PBN. Loss of normal limb morphology is evident in GD 12 embryos treated with thalidomide where the outer most portion of the limb is not uniformly developed. Arrows denote regions where Fgf-10 expression normally occurs.

and GD 12, rabbit embryos treated with thalidomide appeared asymmetrical and malformed. On GD 12 , thalidomide-treated limb buds were notably smaller, and asymmetrical regions of these embryonic limbs coincided with areas of decreased $F g f-10$ expression. Control rabbit embryonic limb buds did not share these same malformations but appeared normal in both size and shape.

Control rabbit limb bud expression of Twist was easily detectable and was uniformly expressed within the normal boundaries of the developing PZ in GD 10 and the established PZ in GD 11 and 12 embryos (Fig. 6). Thalidomide treatment decreased Twist expression in GD 10 rabbit embryonic limbs but, unlike $F g f-10$ expression, was reduced and not entirely eliminated. GD 11 rabbit embryonic limbs showed expression patterns similar to the previous gestational day for which Twist was not inhibited but merely reduced. Twist expression was generally reduced in GD 12 embryonic limbs from thalidomide treatment but showed some areas in the more proximal, lateral aspects where expression was still robust. However, Twist expression in limb buds from GD 12 rabbit embryos was absent in the PZ. Thalidomide-treated rabbit embryonic limbs (GD 11 and 12) were generally smaller and had abnormal morphology. The most distal tip of thalidomide-treated limbs were not round and appeared to be stunted, especially in the area where Twist expression was absent. Rabbit control embryonic limb bud expression of Twist appeared normal.
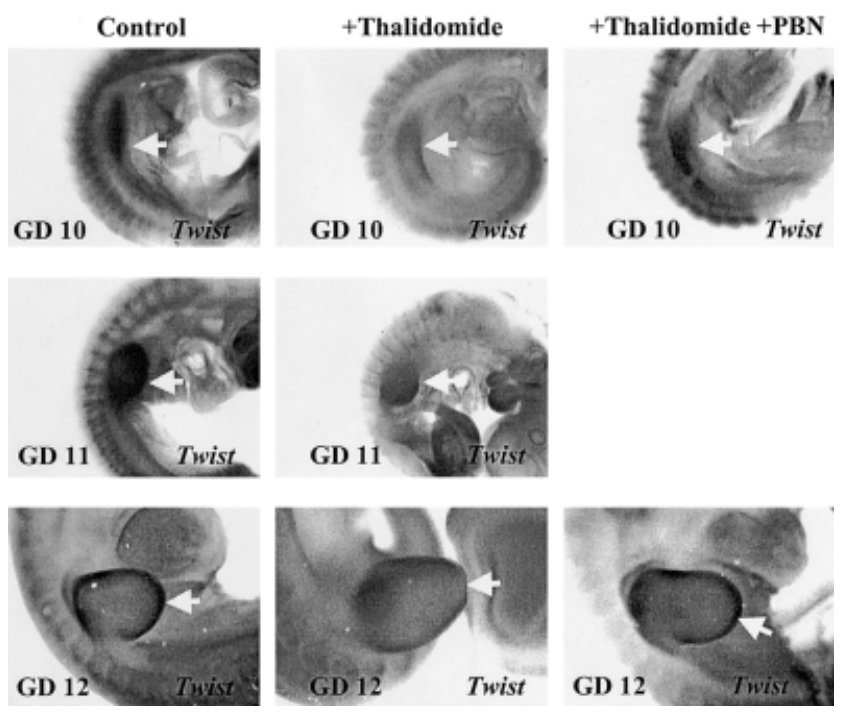

Fig. 6. In situ hybridization of control, thalidomide-treated, and $+\alpha-$ phenyl-N-t-butylnitrone (PBN) rabbit embryos for Twist expression in the gestation day (GD) 10-12 rabbit limb bud. Thalidomide greatly reduced the expression of Twist at each stage of limb development. However, gene expression was not affected in embryos that received both thalidomide and PBN. Loss of normal limb morphology is evident in GD 12 embryos treated with thalidomide where limb bud paddles have failed to develop normally and Twist expression is absent. Arrows denote regions where Twist expression normally occurs.
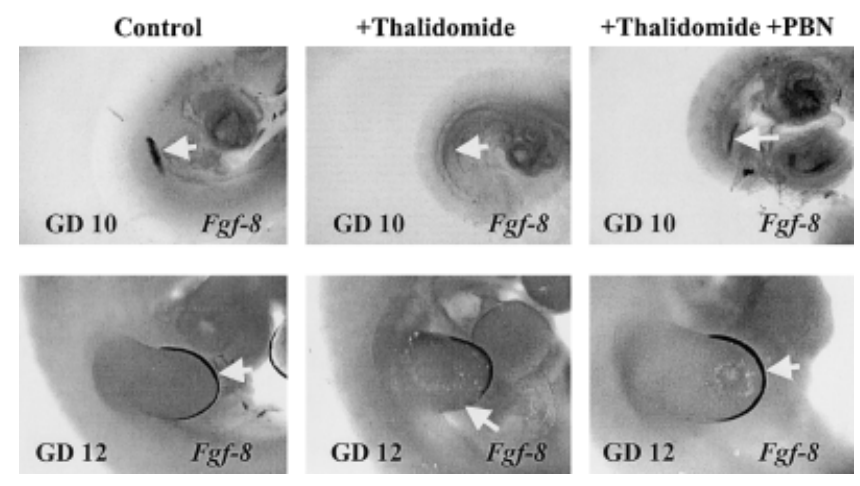

Fig. 7. In situ hybridization of control, thalidomide-treated, and $+\alpha-$ phenyl-N-t-butylnitrone (PBN) rabbit embryos for Fgf-8 expression in the gestation day (GD) 10-12 rabbit limb bud. Thalidomide greatly reduced the expression of Fgf-8 at each stage of limb development. However, gene expression was not affected in embryos that received both thalidomide and PBN. Loss of Fgf-8 expression is most notable in the region of the zone of polarizing activity. Arrows denote regions where Fgf- 8 expression normally occurs.

Control rabbit limb buds from GD 10 embryos showed $F g f-8$ expression and localization in the region of the presumptive AER (Fig. 7) and was even more distinguishable in GD 12 rabbit embryonic limbs. Fgf-8 expression was not detectable in GD 10 rabbit embryonic limbs. GD 12 rabbit embryonic limbs showed normal distribution of $F g f-8$ expression on the anterior portion of the limb only, but $F g f-8$ expression was markedly decreased in the posterior portion of the 
limb. Limb morphology on GD 12 in rabbit embryonic limb buds were relatively symmetrical in areas were Fgf-8 was expressed. However, on the posterior aspect of the developing limb (GD 12), the area of the presumptive ZPA, the limb loses its normal shape, appears to be stunted, and does not share the same symmetry with regions that express $F g f-8$.

Cotreatment with PBN restores the expression of Fgf-10 in the GD 10 rabbit limb buds in the presumptive PZ (Fig. 5). The pattern of $\mathrm{Fg} f-10$ expression in GD 12 rabbit limb buds appeared similar to control limb buds, and expression was reestablished in area of the PZ. The patterns of localization and expression of Twist were also preserved in rabbit limb buds with the cotreatment of thalidomide and PBN (Fig. 6). In rabbit embryos that received both thalidomide and $\mathrm{PBN}$, Fgf-8 expression in the limb bud AER was restored to control patterns of localization and expression of $\mathrm{Fgf-8}$ (Fig. 7). Limb morphology in GD 12 rabbit embryonic limbs was restored and was comparable with control rabbit limb buds.

\section{DISCUSSION}

The disturbances observed in GSH concentration, redox state, and spatial distribution caused by thalidomide exposure are consistent with the type of changes in cellular environment that would result in misregulation of NF- $\mathrm{KB}$-mediated gene expression. $\mathrm{HgO}$ histochemistry identifies regions of GSH localization that show a loss of staining only in the PZ of the sensitive rabbit limb after in utero exposure to thalidomide. The reasons for selective depletion of GSH in the rabbit limb PZ are not entirely understood. Factors related to selectivity may include preferential sequestration or accumulation of thalidomide in PZ cells, increased rates of bioactivation and/or protein binding, or insufficient GSH restoration capacity. Schmahl and coworkers (1996) used radiolabeled EM12, a highly teratogenic thalidomide derivative, and whole-mount autoradiography to show differences in patterns of distribution between the sensitive (marmoset) and resistant (mouse and rat) species. Sensitive species were found to preferentially accumulate EM12 in the limb and neural tube, whereas insensitive species showed no such preferential distribution and deposition. The rabbit may possess similar pharmacokinetic properties that allow for selective accumulation of thalidomide in the limb, although this finding has not been demonstrated and mechanisms have not been described. With regard to species differences in thalidomide metabolism and biotransformation, very little is known about how thalidomide is metabolized or the identity of the proximal reactive species. Arlen and Wells (1996) have suggested that enzymes, such as prostaglandin $\mathrm{H}$ synthase (PHS), may be important in thalidomide-induced oxidative stress, because cotreatment with the irreversible PHS inhibitor, acetylsalicylic acid, in rabbits significantly decreased limb anomalies as well as postpartum fetal lethality. Differences in PHS activity or distribution in the rat and rabbit limbs have yet to be reported.

The PZ region sensitive to GSH depletion by thalidomide is believed to initiate the primary organization and regulation of proximodistal outgrowth of the limb by stimulating the production of growth factors, through the activation and binding of NF-кB. It is also the site for the establishment of the feedback loop between Fgf-10 in the PZ and Fgf-8 in the AER (Crossley et al., 1996; Martin, 1998). Fgf-10 deletions prevent limb outgrowth in mice, resulting in complete amelia, a known effect of thalidomide in humans, and suggesting that Fgf-10 may be involved in thalidomide teratogenesis (Kato and Sekine, 1999).

Thalidomide, which produces an entire spectrum of limb reduction defects in sensitive species, including human, could be interfering with these critical growth pathways. Interference with $\mathrm{NF}-\mathrm{kB}$ activation causes limb defects in both mouse and chick models, for which Twist, Shh, and Fgf-8 expression were down-regulated. These experiments provide the basis for our current understanding of NF-кB's involvement in limb outgrowth pathways (Bushdid et al., 1998; Kangae et al., 1998). TWIST mutations in humans result in selective limb malformations and suggest another possible target for thalidomide-induced loss of gene expression (Paznekas et al., 1998; Rice et al., 2000). Sonic hedgehog $(S h h)$, a critical component of limb patterning, was shown to be affected by the disruption of NF-кB signaling pathways in the limb resulting in complete inhibition of its expression (Bushdid et al., 1998; Kangae et al., 1998). Recent studies where $S h h$ was deleted in a transgenic mouse showed that expression of $F g f-8$ was undetectable in the posterior aspect of the AER, whereas the remainder of the AER was unaffected (Kraus et al., 2001). These same manifestations were evident in rabbit embryos treated with thalidomide (Fig. 7) and suggests a possible relationship between Shh expression and thalidomide-induced limb defects.

To evaluate the effects of thalidomide on NF- $\mathrm{B}$ / DNA binding, transfection of NF-кB-dependent reporters allowed for comparisons in both rat and rabbit LBCs. Other investigators have used electromobility shift assays (EMSA) to determine the effects of different chemicals on NF-кB activation. However, EMSAs

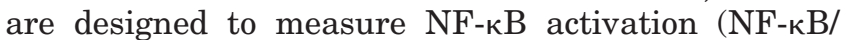
$\mathrm{I}-\mathrm{\kappa} \mathrm{B}$ dissociation) and translocation, and although the assay does measure NF- $\mathrm{B}$ binding, binding occurs under ideal conditions in vitro with isolated nuclear extracts. Drug-induced changes in nuclear redox environment that could alter DNA binding in situ may not be reflected in an EMSA. By using transfection of NF$\kappa \mathrm{B}-$ dependent reporters and cotreating with tetradecanoylphorbol acetate (TPA), to completely activate $\mathrm{NF}-\kappa \mathrm{B}$, we were able to determine effects of thalidomide on NF-кB/DNA binding in intact cells. Diamide, an oxidant and known inhibitor NF-кB /DNA binding, showed a significant decrease in reporter expression in both transfected rat and rabbit LBCs but showed a 
greater effect in the rabbit. Again these differences may be because rabbit conceptuses contain inherently less GSH and cysteine than rat conceptuses (Hansen et al., 1999). Unlike diamide, thalidomide produced an effect only in rabbit LBCs. Diamide effects on NF-кB/ DNA binding correlate with the species that has lower GSH and greater sensitivity to agents that elicit oxidative stress (Hansen et al., 2001b, 2002).

Thalidomide-induced production of ROS can be effectively reversed by the addition of NAC and PBN to the culture media, suggesting that thalidomide (or a metabolite) induces oxidative stress, and it is by oxidative stress that misregulation of NF- $\mathrm{kB} / \mathrm{DNA}$ binding occurs. Previous work has demonstrated the ability of ROS-induced redox potential changes to profoundly effect NF-кB/DNA binding, decreasing NF-кB's ability to successfully transactivate genes (Sun and Oberley, 1996; Galter et al., 1994; Beuerle and Henkle, 1994; Matthews and Hay, 1995; Baldwin, 1996; Sen and Packer, 1996; Flohe et al., 1997; Ginn-Pease and Whisler, 1998).

Other proposed hypotheses of thalidomide teratogenesis do not provide a complete rationale for the incomplete penetrance of thalidomide-induced limb reduction defects, which occur in $30 \%$ of rabbit fetuses with doses of $250 \mathrm{mg} / \mathrm{kg}$ per day on GD 8 and 9 (Schardein, 1993). As a generalization, several factors related to the regulation of intracellular redox status may affect $\mathrm{NF}-\kappa \mathrm{B}$ binding and its dynamic role in regulating gene expression. In the generation of ROS and oxidative stress, treatment with toxicants can result in the reduction of the expression of redox-sensitive dependent genes and, in most cases, does not produce complete inhibition of gene expression. Elimination of ROS and oxidative stress, as observed with cotreatment with NAC and PBN, confirm intracellular redox status to play a critical role in thalidomide-induced modulation of NF- $\mathrm{KB}$ activity. Therefore, thalidomide-induced changes in the redox environment must be sustained and sufficient to decrease the activity of NF-кB to a level where subsequent loss of gene expression compromises the Fgf-10/Fgf- 8 feedback loop and produces limb dysmorphogenesis. The above outlined NF-кB-mediated mechanism can account for both the specific set of terata as well as differences in terata penetrance.

This study outlines a specific mechanism by which limb reduction but not limb patterning is affected by thalidomide. Unlike previously proposed hypotheses, the hypothesis that thalidomide induces redox changes responsible for NF-кB-dependent limb bud outgrowth gene misregulation is consistent across sensitive and insensitive species and provides a solid rationale for common thalidomide-induced terata. In addition, these data suggest that misregulation of other redox-sensitive transcription factors that may serve as a novel mechanism of other teratogens that cause oxidative stress and elicit a distinct set of malformations.

\section{EXPERIMENTAL PROCEDURES}

\section{Reagents}

Thalidomide was purchased from Toronto Research Chemicals (Toronto, Ontario, Canada). HgO, NAC, PBN, and TPA were purchased from Sigma (St. Louis, MO). Hank's balanced salt solution (HBSS) was acquired from Life Sciences (Grand Island, NY). CMRL1066 culturing media was purchased from Gibco Life Technologies (Grand Island, NY). Nu-serum was purchased from Becton-Dickinson (Cincinnati, OH). All other reagents were obtained from common commercial sources.

\section{Animals}

Primagravida Sprague-Dawley rats were obtained from the Toxicology Program Small Animal Facility, University of Michigan (Ann Arbor, MI) on GD 6-9. Day 0 was determined by a sperm-positive vaginal smear on the morning after copulation. Pregnant rats were maintained on a 12-hr light/ 12-hr dark cycle until explantation. Food and water were given ad libitum.

Primagravida New Zealand White rabbits (5.5-7 months old) were purchased from Covance Research Products, Inc. (Kalamazoo, MI). Females were naturally mated with males of the same strain (with the day of copulation assigned as GD 0 at the supplier's facility), shipped on GD 1, and then allowed to acclimate to the new environment until explantation. Food and water were also given ad libitum.

\section{Mercury Orange Histochemistry}

Rabbits were treated between GD 8 and 12 by means of oral gavage with $70 \mathrm{mg}$ thalidomide/kg per day, a dose known to elicit limb reduction defects in New Zealand White rabbit embryos (Schardein, 1993). On GD 12, embryos were removed and fixed in OTC freezing medium (Miles, Inc., Diagnostics Div., Elkhart, $\mathrm{NJ}$ ), snap-frozen in liquid nitrogen, and stored at $-70^{\circ} \mathrm{C}$ until sectioning.

Rats were treated between GD 9-13 by means of oral gavage with $300 \mathrm{mg}$ thalidomide/kg per day. On GD 13, embryos were removed and fixed in OTC freezing medium, snap-frozen in liquid nitrogen, and stored at $-70^{\circ} \mathrm{C}$ until sectioning. Mercury orange staining techniques used are as described by Philbert and coworkers (1991). After treatment with thalidomide in vivo and storage, samples were cryosectioned (10 $\mu \mathrm{m}$ thick), placed on a coverslip, and stained in anhydrous toluene containing mercury orange $(50 \mu \mathrm{M})$ for $5 \mathrm{~min}$. Slides were cleared with two subsequent washes of anhydrous alcohol followed by two washes in dried toluene and mounted in Permount. Slides were stored at $-20^{\circ} \mathrm{C}$ for at least $24 \mathrm{hr}$ before visualization by using fluorescence microscopy at an excitation of $488 \mathrm{~nm}$ and an emission of $530 \mathrm{~nm}$. 


\section{Transfection of Limb Bud Cells With pNF-кB-d2EGFP}

The vector, pNF-кB-d2EGFP (Clontech, Palo Alto, CA), contains four kappa B binding sites in its promoter. Occupation of these sites results in the induction of expression of EGFP, green fluorescent protein. Limb bud cells were isolated from rat (GD 13) and rabbit (GD 12) embryos. On GD 13 in rats and GD 12 in rabbits, embryos were removed from rats and rabbits and placed in warmed HBSS. By using a stereo-dissection microscope, both the forelimbs and hindlimbs were removed from each embryo, and each respective species' limbs were pooled together. Limbs were washed twice in HBSS and placed in a sterile microcentrifuge tube containing calcium/magnesium-free phosphatebuffered saline (CMF-PBS) supplemented with $0.1 \%$ trypsin (w/v) and $0.1 \%$ ethylenediaminetetraacetic acid $(\mathrm{w} / \mathrm{v})$. Samples were placed in a water bath and heated to $37^{\circ} \mathrm{C}$ for $18-20 \mathrm{~min}$ or until cells were sufficiently dissociated. Tubes were inverted two to three times during dissociation and were gently pipetted in and out at the end of the trypsinization to produce a single cell suspension. $\mathrm{Nu}$-serum was added to the suspension to stop the dissociation, and the cells were centrifuged for $5 \mathrm{~min}$ at $1,000 \mathrm{rpm}$ at $5^{\circ} \mathrm{C}$ after which the supernatant was removed and discarded. Cells were resuspended in CMRL-1066 media that had been supplemented with $10 \% \mathrm{Nu}$-serum (v/v) and antibiotics (penicillin and streptomycin). The suspension was filtered by using a $40-\mu \mathrm{m}$ nylon-filtered cell strainer to remove any undissociated cells. Single cells were then resuspended to a final concentration of $2.0 \times 10^{7}$ cells per ml. Aliquots consisting of $1.0 \times 10^{6}$ cells were put into wells containing $3.0 \mathrm{ml}$ of medium in a 6 -well plate. Cultures were maintained in a humidified incubator at $37^{\circ} \mathrm{C}$ in an atmosphere of $95 \% \mathrm{O}_{2}$ per $5 \% \mathrm{CO}_{2}(\mathrm{v} / \mathrm{v})$, allowing the cells to attach to the plate surface. These conditions are found to promote the maintenance and viability of LBCs (Kistler and Howard, 1987). Cells were cultured for 24-48 hr before transfection. pNF-кB-d2EGFP was transfected into LBCs as directed by the supplier of Clonfectin, a liposomal transfection system, purchased from Clontech. A LacZ transfection was also performed to assess transfection efficiency. After transfection (24 $\mathrm{hr})$, cells were treated with thalidomide $(100 \mu \mathrm{M})$ and diamide $(100 \mu \mathrm{M})$, which is known to decrease NF-кB binding (Toledano and Leonard, 1991), as a positive control. Two hours after the initial thalidomide/diamide treatment, cultures were treated with TPA, a phorbol ester that stimulates the dissociation of the $\mathrm{NF}-\kappa \mathrm{B} / \mathrm{I}-\kappa \mathrm{B}$ complex. Some cultures were pretreated (4 hr) with NAC, a precursor to GSH synthesis, or PBN, a free radical trapping agent. After $24 \mathrm{hr}$ of treatment, cultures were washed with PBS and pelleted in microcentrifuge tubes after which the supernatant was removed. The pellet was washed, resuspended, and pelleted two more times in PBS after which it was finally resuspended in sonication buffer $\left(50 \mathrm{mM} \mathrm{NaH} \mathrm{PO}_{4}, 10\right.$
mM Tris-HCl, $200 \mathrm{mM} \mathrm{NaCl}$ [pH 8.0]). Samples were freeze/thawed in a dry ice/ethanol bath five times, vortexed for $1 \mathrm{~min}$, and placed on ice for $1 \mathrm{~min}$. Samples were pipetted in and out of 18-gauge needles several times and then transferred to a microcentrifuge tube and centrifuged for $5 \mathrm{~min}$ at $4^{\circ} \mathrm{C}$. Supernatants were removed and assayed for GFP in a fluorometer at an excitation of $488 \mathrm{~nm}$ and an emission of $510 \mathrm{~nm}$.

\section{In Situ Hybridization}

Probes for mouse $F g f-8$ were provided by Dr. SiewGing Gong (University of Michigan), mouse Fgf-10 by Dr. Gail Martin (University of California, San Francisco, CA), and chick Twist by Dr. Sumihare Noji (University of Tokushima, Japan).

Primagravida rats were treated by gavage with 400 mg thalidomide/kg per day on GD 9 and 10. Embryos were removed on GD 11.5 and 13, fixed in 3.7\% paraformaldehyde in PBS overnight, then prepared for in situ hybridization by washes in methanol.

Primagravida rabbits were treated by gavage with $400 \mathrm{mg}$ thalidomide/kg per day on GD 8 and 9, the sensitive period for limb reduction defects in the New Zealand White rabbit (Fabro, 1981). Embryos were removed on GD 10, 11, and 12 and fixed in 3.7\% paraformaldehyde in PBS overnight then prepared for in situ hybridization by washes in methanol. Further preparation and protocols for whole-mount in situ hybridization that were used in this study are described in detail by Wilkinson (1992).

Some rabbits were pretreated with $40 \mathrm{mg}$ PBN/kg per day by means of intravenous injection before the thalidomide oral gavage doses. Embryos from these rabbits were removed, fixed in $3.7 \%$ paraformaldehyde, and processed for in situ hybridization in an identical manner as those embryos receiving thalidomide only.

\section{ACKNOWLEDGEMENTS}

We thank Rhonda Lightle for her expertise in the mercury orange histology experiments and Chaio Guo for her assistance with in situ hybridization experiments. We also thank the Office of the Vice President of Research, University of Michigan, for their financial support throughout the duration of this project.

\section{REFERENCES}

Arlen RR, Wells PG. 1996. Inhibition of thalidomide teratogenicity by acetylsalicylic acid: evidence for prostaglandin $\mathrm{H}$ synthase-catalyzed bioactivation of thalidomide to a teratogenic reactive intermediate. J Pharmacol Exp Ther 277:1649-1658.

Baldwin AS. 1996. The NF-kB and I-kB proteins. New discoveries and insights. Ann Rev Immunol 14:649-681.

Beuerle PA, Henkle T. 1994. Function and activation of NF-kB in the immune system. Ann Rev Immunol 12:141-179.

Bourgeois P, Bolcato-Bellemin A-L, Danse J-M, Bloch-Zupan A, Yoshiba K, Stoetzel C, Perrin-Schmitt F. 1998. The variable expressivity and incomplete penetrance of the twist-null heterozygous mouse phenotype resemble those of human Saethre-Chotzen syndrome. Hum Mol Genet 7:954-957.

Bushdid PB, Brantley DM, Yull FE, Blaeuer GL, Hoffman LH, Niswander L, Kerr LD. 1998. Inhibition of NF-kB activity results in 
disruption of the apical ectodermal ridge and aberrant limb morphogenesis. Nature 392:615-622.

Crossley PH, Minowada G, MacArthur CA, Martin GR. 1996. Roles for FGF8 in the induction, initiation, and maintenance of chick limb development. Cell 84:127-136.

Fabro S. 1981. The teratogenicity of thalidomide. In: Juchau MR, editor. The biochemical basis of chemical teratogenesis. New York: Elsevier/North-Holland Press.

Flohe L, Brigelius-Flohe P, Saliou C, Traber MG, Packer L. 1997. Redox regulation of NF-kappa B activation. Free Radic Biol Med 22:1115-1126.

Galter D, Mihm S, Droge W. 1994. Distinct effects of glutathione disulphide on the nuclear transcription factor kappa B and the activator protein-1. Eur J Biochem 221:639-648.

Ginn-Pease ME, Whisler RL. 1998. Redox signals and NF-kB activation in T cells. Free Radic Biol Med 25:346-361.

Hansen JM, Carney EW, Harris C. 1999. Thalidomide-induced alterations of glutathione status in organogenesis stage rat and rabbit conceptuses in vitro. Reprod Toxicol 13:547-554.

Hansen JM, Choe H-S, Carney EW, Harris C. 2001a. Differential antioxidant enzyme activities and glutathione content between rat and rabbit conceptuses. Free Radic Biol Med 30:1078-1088.

Hansen JM, Carney EW, Harris C. 2001b. Altered differentiation in rat and rabbit limb bud micromass cultures by glutathione modulating agents. Free Radic Biol Med 31:1582-1592.

Hansen JM, Harris KK, Philbert MA, Harris C. 2002. Preferential depletion of glutathione and alteration of redox status in rabbit vs. rat limbs. J Pharmacol Exp Ther 300:768-776.

Hayashi T, Ueno Y, Okamoto T. 1993. Oxidoreductive regulation of nuclear factor kappa B: involvement of a cellular reducing catalyst thioredoxin. J Biol Chem 268:11380-11388.

Kanegae Y, Tavares AT, Belmonte YC, Verma IM. 1998. Role of Rel/NF-kB transcription factors during the outgrowth of the vertebrate limb. Nature 392:611-614.

Kato S, Sekine K. 1999. FGF-FGFR signaling in vertebrate organogenesis. Cell Mol Biol 45:631-638.

Kistler A, Howard BW. 1987. Testing of retinoids for teratogenicity in vitro: use of micromass limb bud cell culture. Methods Enzymol 190:427-433.

Kraus P, Fraidenraich D, Loomis CA. 2001. Some distal limb structures develop in mice lacking Sonic hedgehog signaling. Mech Dev 100:45-58.

Martin GR. 1998. The roles of FGFs in the early development of vertebrate limbs. Genes Dev 12:1571-1586.

Matthews JR, Hay RT. 1995. Regulation of the DNA binding activity of NF-kB. Int J Biochem Cell Biol 27:865-879.

Matthews JR, Wakasugi N, Virelizier JL, Yodoi J, Hay RT. 1992. Thioredoxin regulates the DNA binding activity of NF- $\mathrm{kB}$ by reduction of a disulphide bond involving cysteine 62. Nucleic Acids Res 20:3821-3830.

Mitomo K, Nakayama K, Fujimoto K, Sun X, Seki S, Yamamoto K. 1994. Two different cellular redox systems regulate the DNA- binding activity of the p50 subunit of NF-кB in vitro. Gene 145:197-203.

Ohuchi H, Nakagawa T, Yamamoto A, Araga A, Ohata T, Ishimaru Y, Yoshioka H, Kuwana T, Nohno T, Yamasaki M, Itoh N, Noji S. 1997. The mesenchymal factor, FGF10, initiates and maintains the outgrowth of the chick limb bud through interaction with FGF8, an apical ectodermal factor. Development 124:2235-2244.

Parman T, Wiley JM, Wells PG. 1999. Free radical-mediated oxidative DNA damage in the mechanism of thalidomide teratogenicity. Nat Med 5:582-585.

Paznekas WA, Cunningham ML, Howard TD, Korf BR, Lipson MH, Grix AW, Feingold M, Goldberg R, Borochowitz Z, Aleck K. 1998. Genetic heterogeneity of Saethre-Chotzen syndrome, due to TWIST and FGFR mutations. Am J Hum Genet 62:1370-1380.

Philbert MA, Beiswanger CM, Waters DK, Reuhl KR, Lowndes HE. 1991. Cellular and regional distribution of reduced glutathione in the nervous system of the rat: histochemical localization by mercury orange and o-phthaldialdehyde-induced histofluorescence. Toxicol Appl Pharmacol 107:215-227.

Rice DP, Åberg T, Chan Y-S, Tang Z, Kettunen PJ, Pakarinen L, Maxson RE, Thesleff I. 2000. Integration of FGF and TWIST in calvarial bone and suture development. Development 127:18451855.

Sauer H, Gunther J, Heschler J, Wartenberg M. 2000. Thalidomide inhibits angiogenesis in embryoid bodies by the generation of hydroxyl radicals. Am J Pathol 156:151-158.

Schardein JL. 1993. Chemically induced birth defects. New York: Marcel Dekker, Inc.

Schmahl H-J, Dencker L, Plum C, Chahoud I, Nau H. 1996. Stereoselective distribution of the teratogenic thalidomide analogue EM12 in the early embryo of marmoset monkey, Wistar rat and NMRI mouse. Arch Toxicol 70:749-756.

Sen CK, Packer L. 1996. Antioxidant and redox regulation of gene transcription. FASEB J 10:709-720.

Summerbell D. 1974. A quantitative analysis of the effect of excision of the AER from the chick limb-bud. J Embryol Exp Morphol 32: $651-660$.

Sun Y, Oberley LW. 1996. Redox regulation of transcriptional activators. Free Radic Biol Med 21:335-348.

Toledano M, Leonard WJ. 1991. Modulation of transcription factor NF- $\mathrm{KB}$ binding activity by oxidation-reduction in vitro. Proc Nat Acad Sci USA 88:4328-4332.

Vogel A, Rodriguez C, Izpisúa-Belmonte J. 1996. Involvement of FGF-8 in initiation, outgrowth and patterning of the vertebrate limb. Development 122:1737-1750.

Wilkinson DG. 1992. Whole mount in situ hybridization of vertebrate embryos. In: In situ hybridization. Oxford: Oxford University Press. $75 \mathrm{p}$.

Xu X, Weinstein M, Li C, Naski M, Cohen RI, Ornitz, DM, Leder P, Deng C. 1998. Fibroblast growth factor receptor 2 (FGFR2)-mediated reciprocal regulation loop between FGF8 and FGF10 is essential for limb induction. Development 125:753-765. 